gales of force 7 and over. The charts would also depict currents, great-circle tracks, loadline zones, ice limits, sea temperature and air humidity.

When the first four sheets have been compiled, the Hydrographic Department of the Admiralty have indicated their intention to inform the Chart Users' Advisory Panel with a view to discussing the display of information before printing.

\title{
Interpreting Astro-position Lines at Sea
}

$$
\text { from M. W. Richey }
$$

'THE mark of a good navigator is not so much his ability to obtain accurate information as his ability to evaluate and interpret correctly the information available to him.' So writes Captain Alton B. Moody, U.S.N.R. in the course of an interesting correspondence in the February number of the U.S. Naval Institute Proceedings. In the August 196 I number (page 132) Captain Robert Lee Rhoads, Jr., a shipmaster, suggests that the 'cartwheel' set of astro-sights, where four position-lines intersect each other at angles of about $45^{\circ}$, is inferior to the square where the position-lines intersect at approximately $90^{\circ}$, which gives a more reliable fix. One of Captain Rhoads's illustrations shows a case of a 4 -minute systematic error in a 'cartwheel' fix giving a position 6 miles in error, where the same error would have had no effect on the position interpolated from four sights taken at $90^{\circ}$ to each other.

In the February number, Commander Roger S. Strout, U.S.N.R., refers to Rear Admiral L. Tonta's paper in the Hydrographic Review for November 193 I, in which he describes the bisector method of analysing the plot. Tonta points out that systematic errors, which arise particularly from anomalies of the dip of the horizon, can assume large values and their certain elimination is the precondition of accurate fixing.

Commander Strout points out that the essential difference between Captain Rhoads's method of interpolation between position-lines where the pattern is opened out through systematic errors, and the one described by Admiral Tonta, is that the latter adds an arrow to each position-line pointing towards the star. Commander Strout goes on to say that the bisector method is too slow and messy for practical use on the plotting sheet, and suggests that since only one point on a bisector is normally used, an equal-distance method in which the observed position is assumed to be at an equal distance from each altitude position-line, can be substituted without loss of accuracy, and with a great gain in speed and clarity. Marking the arrows on the position-lines also enables the good sights to be distinguished from the bad ones when four sights are used. With three sights, blunders cannot be distinguished from systematic errors, but the study of a fourline plot will often reveal a blunder, though neither the magnitude nor the direction of the error will be indicated. With more than four sights-and Commander Strout suggests that a careful observer usually takes six-a blunder 
is readily identified. The plot shown in Fig. I, for example, appears hopeless at first, but the arrows indicate a correction towards the stars, and a few seconds with the dividers will yield the quite acceptable position shown. Five of the position-lines agree to within a mile of the observed position.

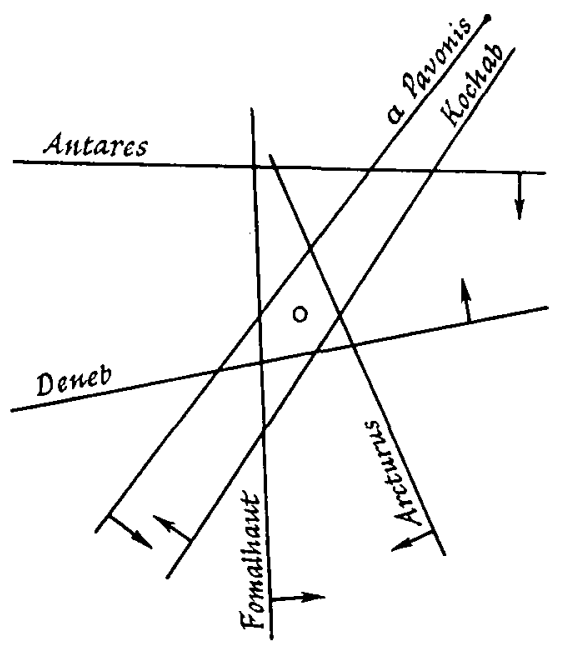

FIG. I

In the same number (February 1962), Commander Sobieralski, of the U.S. Coast and Geodetic Survey, points out that a three-line fix with azimuths differing by approximately $120^{\circ}$ can be just as useful as a fourline fix in eliminating the effect of a constant altitude error on all sights, since the centre of the triangle will be the same whether the altitudes are too large or too small. In the Coast and Geodetic Survey, navigating officers use the device of drawing in a small arrow towards the star on each position-line. In this way, with a 'cartwheel' fix, it becomes a simple matter to locate the alternative positions and select the most probable one.

Captain Alton B. Moody, in the same number, describes what he terms 'the better known technique' of bisecting the external angle (the angle between the azimuth lines at each intersection of lines of position) and using the intersection of the bisectors as the fix. The additional work involved in bisecting angles can be avoided by observing three or more bodies equally spaced in azimuth around the entire horizon. A possible advantage, he says, of observing four stars $90^{\circ}$ apart over three stars $120^{\circ}$ apart, is that the relative spacing of the two sets of reciprocal lines of position of a four-star set, might provide some indication of the magnitude of the constant error and the probable size of the random error. Captain Moody ends by regretting that the rather voluminous literature which has accumulated on the subject of navigational errors, should appear to have made so little impact on navigational practice.

The use of bisectors was fully described in a paper by Commander M. Bini published in the July 1955 number of this Journal. Bini concludes that the threeline fix should never be accepted as the standard method of determining position astronomically at sea, since the plot can give no indication from its shape of what is wrong. The use of four position-lines enables the observer to judge whether the observations are affected by a non-systematic error, which, he suggests, is the only one that matters, since systematic errors can be eliminated by the use of bisectors for analysing the plot. The ideal, he suggests, is to observe five stars, but only to work up the fifth when it is necessary to identify a blunder.

The bisector, which is in fact a position-line obtained from equal differences of altitude, is independent of systematic error. The best bisector will be derived from sights separated in azimuth by $180^{\circ}$ but, because of the slow variation of the sine curve, the optimum conditions can in practice be extended to $15^{\circ}$; it is not advisable to use bisectors where the difference of azimuth is less than $60^{\circ}$. Between the limits of $\mathrm{I} 50-180^{\circ}$, besides eliminating systematic error, the bisector 
method averages random errors. A more reliable position will thus be derived from the intersection of bisectors than the intersection of altitude position-lines.

Bini suggests that the whole practice of astronomical navigation at sea might appear in a new light if some of the time saved by the improvement in almanacs and tables, were devoted to the analysis of the observations and plot. His paper (and the relevant sections of his book Navigazione Astronomica), even though they describe well-known principles, make a very practical contribution to the problem of evaluating astronomical observations at sea.

A suggestion, due to $\mathrm{Mr}$. D. H. Sadler, for recognizing a blunder in a set of four position-lines at right angles, without having to draw in the arrows on each position-line, is the simple one of deliberately introducing a constant systematic error into all the observations (or alternatively, ignoring the dip). This would have the effect of opening out the pattern and making certain that, apart from blunders, the true position lies within the pattern. If the position-lines are reasonably spaced, then the constant error will be taken out.

In Volume 5 of the Journal, J. B. Parker gives a mathematical treatment of the problem of analysing simultaneous positional data in the air, and so deliberately ignores systematic error which (unlike the case of sea navigation) can in principle be reduced to insignificance by careful calibration of instruments. The paper shows how to detect blunders, using the size of the cocked hat formed by three position-lines as a criterion, and justifies the use of the in-centre as the most probable position if the blunder criterion is not met. These procedures are based on the assumption that, apart from possible blunder, the only errors are random ones distributed in a Gaussian manner. In a further note contributed to the Forum $(\mathbf{1 4}, 473)$ Parker, commenting on an article by Captain Cotter on 'The Cocked Hat' ( $\left.\mathbf{r}_{4}, 223\right)$, concludes that three pieces of information do not throw a great deal of light on position when both systematic and random errors and possibly blunders as well, may occur.

So far as navigation at sea is concerned, what seems to be required is a standard procedure for analysing the plot which will enable the navigator both to detect blunders and to derive the most probable position from a set of altitude positionlines. Hydrographic surveyors in the Royal Navy, apparently, determine position from a set of sights by the same methods when navigating the ship as when surveying. These involve drawing in the azimuth arrows which, unless there is abnormal dip or refraction, should all point towards or away from the centre of the figure described by the position-lines. The centre of the circle of best fit (in which the residuals are all roughly equal) is accepted as the most probable position. It is strange that the use of this or a similar safeguard against misinterpreting astro-position lines (such as the bisector method) is not normally enjoined upon navigating officers in the Royal Navy—or indeed outside it.

\section{RE F E R E N C ES}

Tonta, L. (193I). Accurate determination of the position at sea. Hydr. Rev., 8, 33. Anderson, E. W. (1952). The treatment of navigational errors. This Journal, $5,103$.

Parker, J. B. (1952). The treatment of simultaneous position data in the air. This Journal, $5,235$.

Bini, M. (1955). The use of bisectors in selecting the most probable position. This Journal, 8, 195 .

Anderson, E. W. and Parker, J. B. (1 956). Observational errors. This Journal, 9, 105. 